\title{
Culex quinquefasciatus vitellogenesis: morphological and biochemical aspects
}

\author{
André F Cardoso, Renato L Cres, Alexandre S Moura, Fábio de Almeida, A Tania Bijovsky/+
}

Departamento de Parasitologia, Instituto de Ciências Biomédicas, Universidade de São Paulo, 05508-900 São Paulo, SP, Brasil

The vitellogenic process in Culex quinquefasciatus, which is triggered by a blood meal, involves the synthesis, distribution and storage of the nutrients necessary for embryo development. The fat body of an adult female Cx. quinquefasciatus revealed two cell types: large trophocytes and small, eosinophilic, "oenocyte-like" cells, which show no morphological changes throughout the gonotrophic cycle. Trophocytes, which only begin to synthesise vitellogenin (Vg) $12 \mathrm{~h}$ post-blood meal (PBM), undergo a series of morphological changes following engorgement. These changes include the expansion of the rough endoplasmic reticulum (RER) and Golgi complex, which are later destroyed by autophagosomes. At 84 h PBM, trophocytes return to their pre-engorgement morphology. The ovarian follicles of non-blood-fed Cx. quinquefasciatus contain a cluster of eight undifferentiated cells surrounded by follicular epithelium. After engorgement, the oocyte membrane facing the perioocytic space increases its absorptive surface by microvilli development; large amounts of Vg and lipids are stored between 24 and $48 \mathrm{~h}$ PBM. Along with yolk storage in the oocyte, follicular cells exhibit the development of RER cisternae and electron-dense granules begin to fill the perioocytic space, possibly giving rise to endochorion. Later in the gonotrophic cycle, electron-dense vesicles, which are possible exochorion precursors, fuse at the apical membrane of follicular cells. This fusion is followed by follicular cell degeneration.

Key words: Culex quinquefasciatus - vitellogenesis - immunolabelling - morphology - fat body - ovary

During the course of oogenesis, insects accumulate large amounts of resources inside their eggs in the form of proteins, lipids, carbohydrates and other minor components. These supplies or "yolk" are critical for embryonic development and are produced in both autosynthetic and heterosynthetic manners (Tadkowski \& Jones 1978, de Bianchi et al. 1985, Raikhel \& Dhadialla 1992, Attardo et al. 2005). Yolk formation in mosquitoes has been extensively studied in Aedes aegypti (Raikhel 1984, 1987, Dhadialla \& Raikhel 1990, Snigirevskaya et al. 1997), a species that has become the model for mosquito oogenesis.

The insect fat body is a multifunctional organ involved in intermediary metabolism and the storage of fat, glycogen and protein (Chapman 1998). Trophocytes are the main cell type of fat body tissue. Their cytoplasm is mainly filled with lipid droplets, while the remaining space is occupied by the nucleus and a thin perinuclear strip containing a few organelles such as rough endoplasmic reticulum (RER), Golgi complex, mitochondria and lysosomes.

The sequence of ultrastructural changes occurring in the trophocytes of vitellogenic Ae. aegypti, includes the prominent expansion of the RER and Golgi complex, and

Financial support: FAPESP, CAPES

+ Corresponding author: bijovsky@icb.usp.br

Received 22 September 2009

Accepted 25 February 2010 an increase in the number of mitochondria (Raikhel \& Lea 1983). In Ae. aegypti, vitellogenin (Vg), the most abundant yolk protein precursor, is synthesised exclusively in fat body cells (Hagedorn \& Judson 1972, Hagedorn et al. 1973), secreted into the haemolymph and internalised by receptor-mediated endocytosis by developing oocytes (Snigirevskaya et al. 1997, Sappington \& Raikhel 1998). At the end of vitellogenesis, fat body cells are characterised by activation of the lysosomal machinery with the formation of numerous autophagosomes (Raikhel \& Lea 1983, Raikhel 1986, Snigirevskaya et al. 1997).

Mosquito ovaries are dorsolaterally situated in the posterior region of the abdomen and each is composed of functional units called ovarioles (Clements 1992). Each ovariole contains a germarium, where germ cells proliferate and follicles are formed, and a vitellarium, where oocytes develop and undergo the storage of yolk proteins. During each gonotrophic cycle, the follicles within each ovariole mature synchronously. Each follicle is surrounded by a single layer of follicular cells, which are derived from somatic tissue and will secrete components of the eggshell (Clements 1992, Raikhel \& Dhadialla 1992).

Culex quinquefasciatus is a cosmopolitan mosquito that is highly anthropophilic and completely adapted to urban conditions with a larval stage capable of developing in highly polluted water collections. This buzzing and biting mosquito is responsible for great nocturne discomfort and allergic responses (Malafronte et al. 2003). Additionally, Cx. quinquefasciatus transmits pathogens such as Wuchereria bancrofti, one of the agents of lymphatic filariasis and is a competent vector of neurotropic viruses (Gaunt et al. 2001). The St. Louis 
and Japanese encephalitis viruses, eastern and western equine encephalomyelitis viruses, Rift Valley virus and West Nile virus are efficiently transmitted by $C x$. quinquefasciatus (Vaidyanathan \& Scott 2007, Hamer et al. 2008). Therefore, it is important to control the populations of this mosquito and the transmission of its associated pathogens.

Although vector mosquitoes of the Aedes, Anopheles and Culex genera share many developmental, biochemical, behavioural and morphological traits, significant differences do occur. For example, $C x$. quinquefasciatus diverges from mosquitoes of other genera, i.e., Aedes and Anopheles, in the morphology of its salivary glands and saliva composition (da Cunha Sais et al. 2003, Ribeiro et al. 2004) in the cellular and biochemical processes involved with blood digestion and haem detoxification (Okuda et al. 2002, 2007) and in its response to odorants and biting behaviour (Van Essen et al. 1994). Therefore, comparative studies may provide a basis for the development of novel strategies and the improvement of the current approaches for vector control.

In this paper, we describe the changes in the fat body and ovaries of $C x$. quinquefasciatus during the first gonotrophic cycle.

\section{MATERIALS AND METHODS}

Animals - Cx. quinquefasciatus (PIN strain) mosquitoes were raised at $26^{\circ} \mathrm{C}$ in $70-80 \%$ relative humidity and a photoperiod of $12 \mathrm{~h}$ dark- $12 \mathrm{~h}$ light. Adults were fed a $10 \%$ sucrose solution ad libitum. When necessary, 5-7-day-old adult females were fed on Balb/c mice anaesthetised with $0.3 \mathrm{mg} / \mathrm{kg}$ of xylazine hydrochloride (Calmiun, Agner União) plus $30 \mu \mathrm{g} / \mathrm{kg}$ of acepromazine (Acepran, Univet SA, São Paulo, Brazil).

Gel electrophoresis - Ovaries and fat bodies (i.e., abdomens free of gut, Malpighian tubules and ovaries) (Fallon et al. 1974) were dissected before engorgement and at $3,6,9,12,24,48,72$ or $96 \mathrm{~h}$ post-blood meal (PBM). They were then homogenised in PBS containing $1 \mu \mathrm{l} / \mathrm{mL}$ of a cocktail of protease inhibitors $(50 \mu \mathrm{g} / \mathrm{mL}$ leupeptin, $5 \mu \mathrm{g} / \mathrm{mL}$ pepstatin, $5 \mu \mathrm{g} / \mathrm{mLchymostatin}$, $5 \mu \mathrm{g} / \mathrm{mL}$ antipain, $5 \mu \mathrm{g} / \mathrm{mL}$ PMSF) and $5 \mu \mathrm{M} \mathrm{E}-64$; the protein content was determined using the Bradford assay (Bradford 1976). SDS-PAGE was performed on 8\% polyacrylamide gels (Laemmli 1970) and proteins were visualised by staining with Coomassie Brilliant Blue R-250. Molecular masses were estimated using protein standards (Bio-Rad Laboratories).

Antibody production - Monospecific polyclonal antisera against yolk protein were raised in Balb/c mice. $C x$. quinquefasciatus ovaries (48 h PBM) were subjected to SDS-PAGE. After electrophoresis, the Coomassie bluestained bands corresponding to the 86 and $192 \mathrm{kDa}$ vitellin fractions were excised from the gel and minced the products were used independently for two intraperitoneal immunisations with an interval of 15 days between them. Seven days after the second immunisation, the animals were bled to obtain the sera.
Immunoblotting - Proteins subjected to SDS-PAGE were electroblotted onto a nitrocellulose membrane. The membrane was blocked overnight with Tris-buffered saline [TBS; $0.02 \mathrm{M}$ Tris(hydroxymethyl)aminomethane, $0.15 \mathrm{M} \mathrm{NaCl}, \mathrm{pH} 8.2] \mathrm{pH} 7.4$ containing $5 \%$ non-fat milk (blocking buffer). Membrane strips were incubated with a 1:600 dilution of both anti-vitellin sera (anti-vg, small fraction; anti-VG, larger fraction) in blocking buffer for 90 min with agitation. Mouse pre-immune serum was used as a control. After three serial washes (5 min each) in TBS $\mathrm{pH} 7.4$, the bound antibodies were detected with peroxidase-conjugated goat anti-mouse polyvalent immunoglobulins (Cappel) diluted 1:1,500 in blocking buffer. After another set of washes, the immunoblots were developed by the addition of $\mathrm{H}_{2} \mathrm{O}_{2}$ and 4-Chloro1-Naphthol. The reactions were stopped by transferring the membrane to deionised water. Fig. 1 shows the specificity of the antisera with each recognising a single band of the expected mass ( 86 and $192 \mathrm{kDa}$ ).

Morphology - Five insects were used for each analysis, which was performed before engorgement and at 6 , $12,18,24,36,48,60,72,84$ and 96 h PBM. Fat bodies and ovaries were dissected in PBS under a stereoscopic microscope.

Fat bodies were fixed for $2 \mathrm{~h}$ at $4^{\circ} \mathrm{C}$ in $3.5 \%$ glutaraldehyde in $0.1 \mathrm{M}$ phosphate buffer, $\mathrm{pH}$ 7.2. Following post-fixation with $1 \%$ osmium tetroxide, the samples were dehydrated in a graded ethanol series.

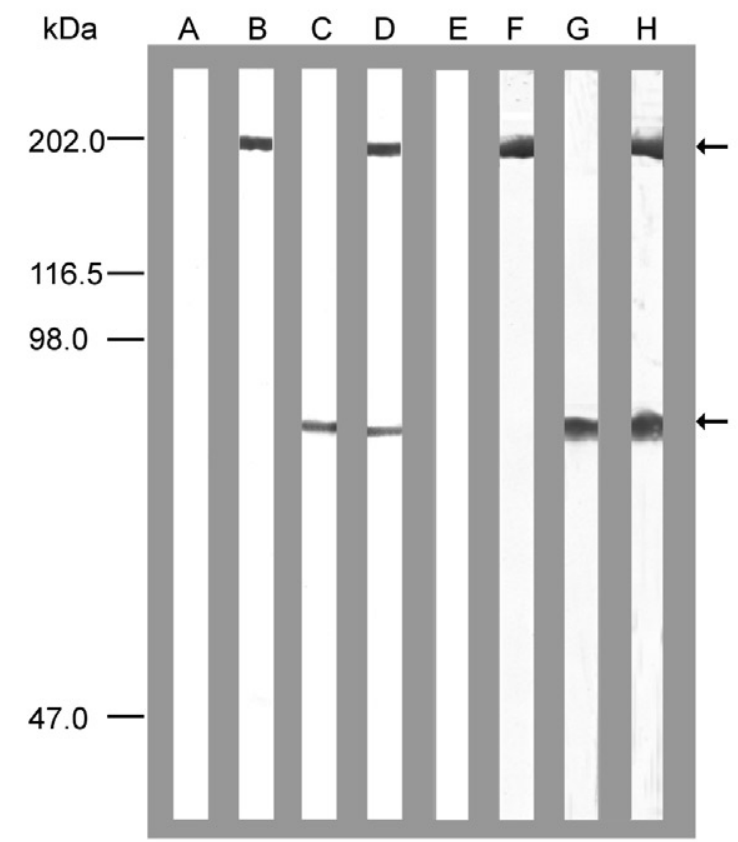

Fig. 1: immunoblot of fat body (A-D) and ovary (E-F) extracts. Both antibodies are highly specific each recognizing a single band. Ar rows indicate the large $(192 \mathrm{kDa})$ and small $(86 \mathrm{kDa})$ polypeptides of vitellogenin (Vg). A: pre-immune serum; B: anti-VG; C: anti-vg; D: anti-VG plus anti-vg; E: pre-immune serum; F: anti-VG; G: anti-vg; $\mathrm{H}$ : anti-VG plus anti-vg. 

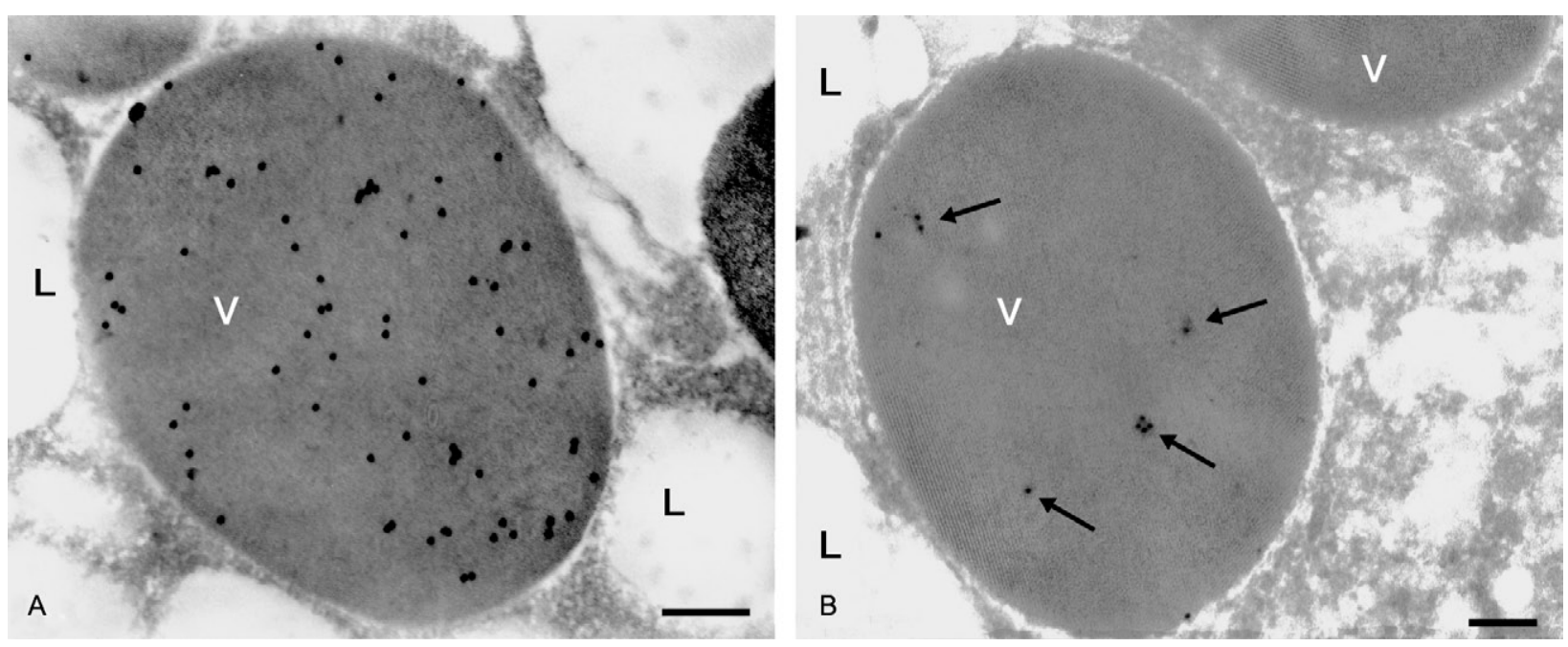

Fig. 2: immunocytochemistry of ovaries of $24 \mathrm{~h}$ post-blood meal Culex quinquefasciatus using the policlonal antibodies anti-vg (A) and anti-VG (B, arrows). Note the high affinity of each antibody and the low sensibility of anti-VG in this system. L: lipid droplets; V: vitellin granules. Bar: $200 \mathrm{~nm}$.

Ovaries were fixed for $2 \mathrm{~h}$ in $0.5 \%$ glutaraldehyde and $4 \%$ paraformaldehyde in $0.1 \mathrm{M}$ sodium cacodylate buffer $\mathrm{pH} 7.2$ at RT. After post-fixation with $1 \%$ osmium tetroxide, samples were contrasted en bloc with $2 \%$ uranyl acetate in $70 \%$ acetone and dehydrated in a graded acetone series. After dehydration, the samples were embedded in Spurr's resin. Semithin sections (200-300 nm) were stained with toluidine blue for light microscopy studies. Ultrathin sections $(70-80 \mathrm{~nm})$ were stained with uranyl acetate and lead citrate and studied at $80 \mathrm{kV}$ in a JEOL 100CX transmission electron microscope.

Immunocytochemistry - Fragments of Cx. quinquefasciatus ovaries $48 \mathrm{~h}$ PBM were fixed for $1 \mathrm{~h}$ in $0.5 \%$ glutaraldehyde, $4 \%$ paraformaldehyde and $0.2 \%$ picric acid in $0.1 \mathrm{M}$ cacodylate buffer ( $\mathrm{pH}$ 7.2) containing $1 \mathrm{mM}$ $\mathrm{CaCl}_{2}$. After fixation, the fragments were embedded in LR White and placed in gelatin capsules for polymerisation at $37^{\circ} \mathrm{C}$. Silver sections placed onto nickel grids were washed in TBS and incubated as described by Okuda et al. (1999). Fig. 2A, B illustrates the specific labelling of the vitellin granules. Due to the higher sensitivity of the anti-vg antibody in immunocytochemistry, this antibody was also chosen for the immunoblot experiments.

Ethics - All procedures involving mice manipulation were approved by the Ethical Committee on Animal Experiments of the Institute of Biomedical Sciences of São Paulo University.

\section{RESULTS}

The fat body - As described in other insects, the fat body of $C x$. quinquefasciatus is organised into a sheet of cells distributed beneath the body wall, suspended in the haemolymph and involving the viscera (Fig. 3A).

Before engorgement, light microscopy of the $C x$. quinquefasciatus fat body revealed two cell types. Most of the organ is composed of large trophocytes with a highly vacuolated cytoplasm. Mingled with them are small cells characterised by their eosinophilic cytoplasm (oenocyte-like cells) and occurring in isolation or in small groups (Fig. 3A). At the ultrastructural level, oenocyte-like cells display a dense cytoplasm containing numerous mitochondria with noticeable cristae and electron-dense granules; the nuclei show a prominent nucleolus and abundant peripheral heterochromatin (Fig. 3B). Oenocyte-like cells showed no ultrastructural changes throughout the first gonotrophic cycle. These cells not appear to be involved in the changes in the protein profile observed in Fig. 4A, C.

The trophocytes of non-blood-fed females are large cells with a cytoplasm that is almost entirely occupied by conspicuous lipid droplets and some glycogen depots. A thin ring of poorly developed cytoplasm surrounds a spherical nucleus with a prominent nucleolus and dispersed heterochromatin (Fig. 5A). Scarce, small cisternae of the RER are found at $6 \mathrm{~h}$ PBM, usually in the vicinity of the nucleus (Fig. 5A, insert). At 12 h PBM, they are already easily noticeable and some of them are spiralled. RER cisternae continue to increase in number and, together with Golgi cisternae, nearly fill the perinuclear cytoplasm at $24 \mathrm{~h}$ PBM (Fig. 5B). At this point, the electron-dense vesicles and granules emerging from the Golgi complex are also evident (Fig. 5C). Only $12 \mathrm{~h}$ after a blood meal, the presence of $\mathrm{Vg}$ is noticeable in the fat body extracts (Fig. 4A, C), which is consistent with the scarce development of RER observed at the previous times (Fig. 5A, insert). Vg remains detectable until $48 \mathrm{~h}$ PBM. At that time, the biosynthetic organelles begin to decline and autophagosomes are observed between 48$72 \mathrm{~h}$ PBM (Fig. 5D, E). Only at $84 \mathrm{~h}$ PBM do the trophocytes return to the appearancethat they had before the blood meal (Fig. 5F). 

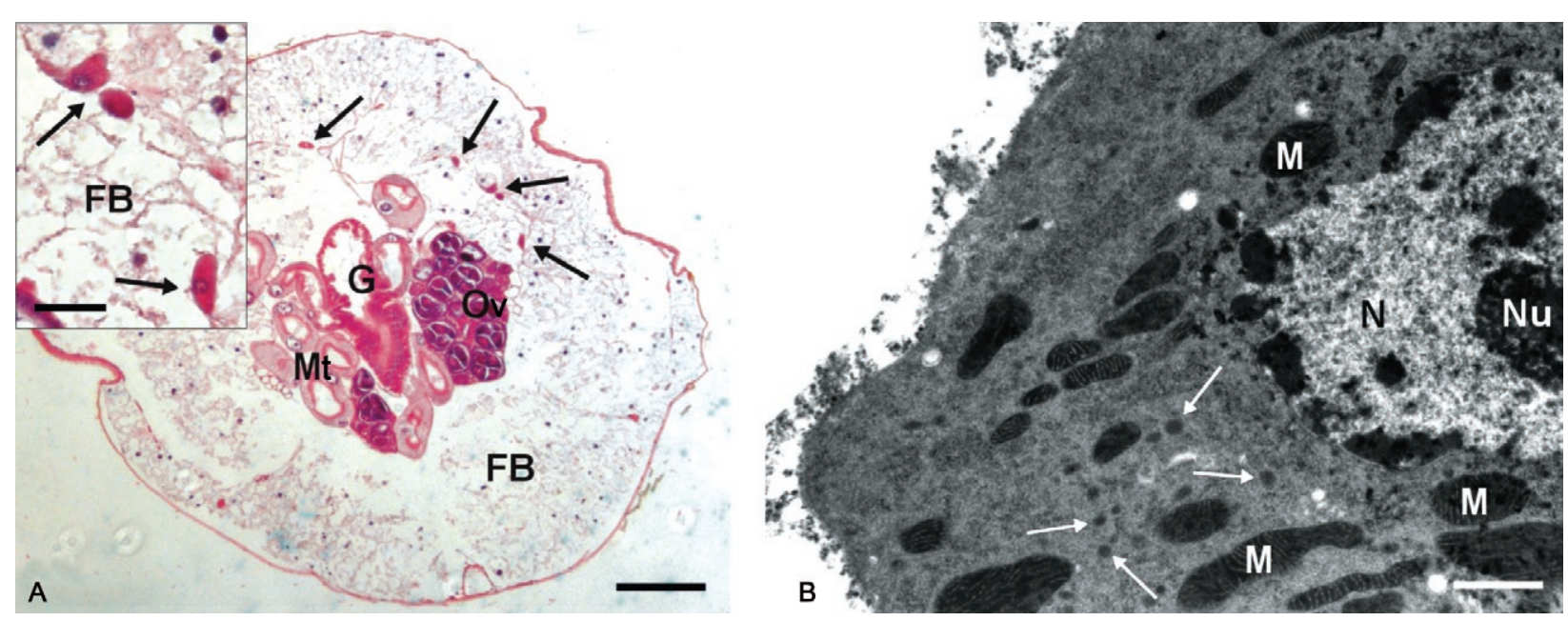

Fig. 3: Culex quinquefasciatus 7-days-old non blood-fed female. A: light micrograph of a haematoxylin-eosin stained cross-section through mosquito abdomen. Ovaries (Ov), midgut $(\mathrm{G})$ and Malphigian tubules (Mt) are surrounded by fat body cells (FB), which fill all the space beneath the cuticle. Arrows point oenocyte-like cells, enlarged in the insert. Bar: $50 \mu \mathrm{m}$, insert: $10 \mu \mathrm{m}$; B: electron micrograph of an oenocyte-like cell with characteristic electrondense cytoplasm containing abundant mitochondria (M) and tiny electrondense granules (arrows). N: nucleus; Nu: nucleolus. Bar: $1 \mu \mathrm{m}$.
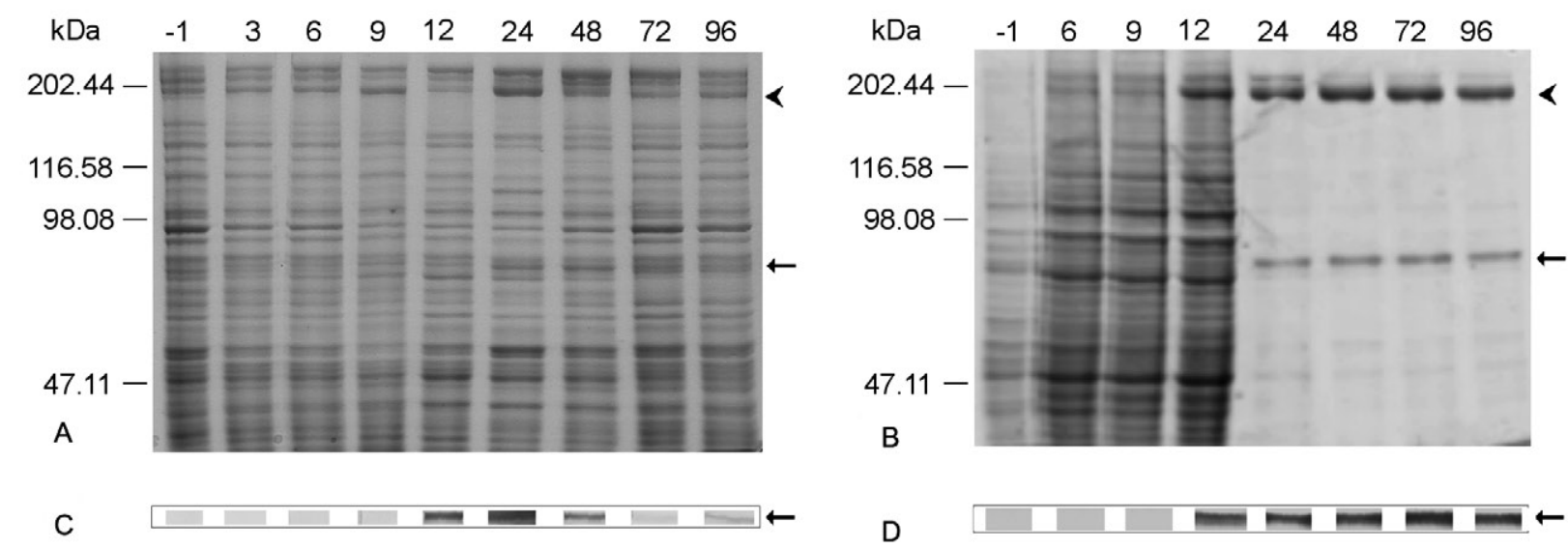

Fig. 4: protein profile analysis of fat bodies (A) and ovaries (B) of Culex quinquefasciatus after blood meal. Vitellogenin (Vg) olypeptides of around 192 (arrowheads) and 86 (arrows) kDa were observed only after blood meal. The numbers above lanes indicate hours post blood meal. Immunoblot (anti-vg, arrows) of fat bodies (C) and ovaries (D) of Cx. quinquefasciatus. kDa: molecular weight.

The ovaries - Ultrastructure of pre-engorged Cx. quinquefasciatus ovaries reveals follicles formed by a set of undifferentiated cells, several of them exhibiting prominent nucleolus. This set of cells is surrounded by a monolayer of flattened cells with dense cytoplasm, the follicular epithelium (Fig. 6A), which does not display any morphological sign of synthetic activity (endoplasmic reticulum and/or Golgi cisternae).

Only after engorgement can the oocyte be distinguished from the remaining cells that give rise to the nurse cells. Soon after a blood meal, the oocytes develop microvilli on their plasma membranes (Fig. 6B, C), which border on the perioocytic space (between the follicular cells and the oocyte). Coated vesicles are noticeable in the peripheral cytoplasm (Fig. 6B, insert). The accumulation of Vg in the ovary extracts starts to be noticeable around $12 \mathrm{~h}$ PBM (Fig. 4B, D). Concomitantly, the formation of vitellin granules and the accumulation of lipid droplets are observed (Fig. 6B). During the subsequent developmental stages, $C x$. quinquefasciatus oocytes undergo few morphological changes besides their obvious growth. The storage of vitellin and lipids continues and at $24 \mathrm{~h}$ PBM the yolk granules occupy almost the entire cytoplasm (Fig. 6C, insert). Only at $96 \mathrm{~h}$ PBM does glycogen begin to accumulate (Fig. 6F). 

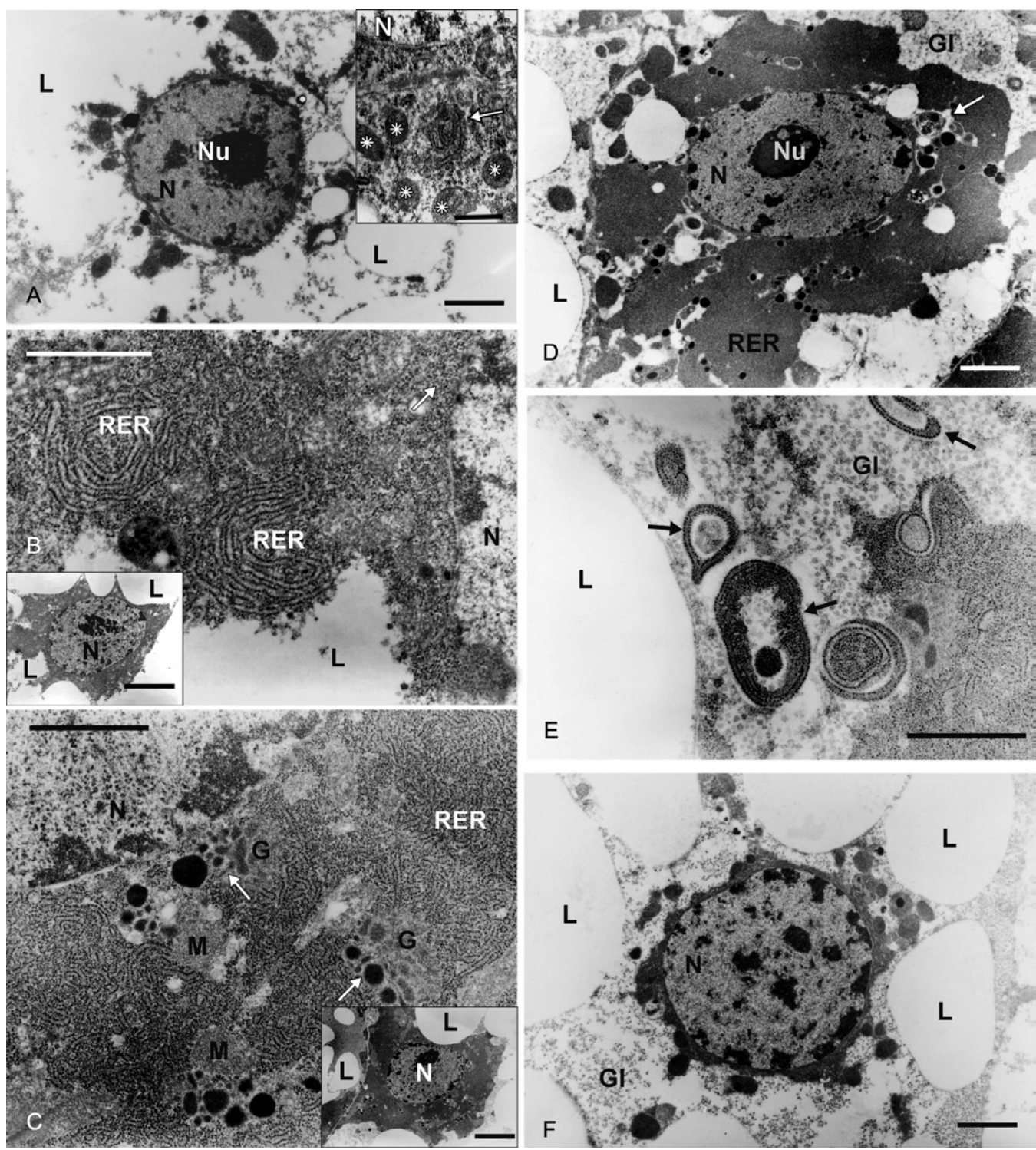

Fig. 5: electron micrographs of fat body of Culex quinquefasciatus. A: typical trophocyte of a 7-days-old non blood-fed female with its cytoplasm almost entirely occupied by lipid droplets (L). Nu: nucleolus (Bar: $2 \mu \mathrm{m}$ ). The insert shows a $6 \mathrm{~h}$ post-blood meal (PBM) trophocyte. Small rough endoplasmic reticulum (RER) cisterna of circular profile (arrow) is seen at the nucleus (N) neighbouring. Asterisks mean mitochondria (M). Bar: $500 \mathrm{~nm}$; B: $12 \mathrm{~h}$ PBM trophocyte. Observe the circular profiles of the cisternae (RER). Bar: $500 \mathrm{~nm}$. The insert shows the already developed cytoplasm around the N. Bar: $4 \mu \mathrm{m}$; C: $24 \mathrm{~h}$ PBM trophocyte. Insert shows the well developed perinuclear cytoplasm that, besides to numerous RER cisternae, exhibits transport vesicles (arrows) in association with G. Bar: $1 \mu \mathrm{m}$. Insert: $2 \mu \mathrm{m}$; D: $48 \mathrm{~h}$ PBM trophocyte. Perinuclear cytoplasm begins to present autophagosomes (arrow). Gl: glycogen. Bar: $2 \mu \mathrm{m}$; E: $48 \mathrm{~h} \mathrm{PBM}$ trophocyte. Presence of numerous autophagosomes (arrows) indicating the degradation of the synthetic machinery. Bar: $1 \mu \mathrm{m}$ : F: $84 \mathrm{~h}$ PBM trophocyte. The perinuclear cytoplasm has returned to the same characteristics of non-blood fed mosquito. Bar: $2 \mu \mathrm{m}$.

The follicular cells begin to display RER cisternae at approximately $24 \mathrm{~h}$ PBM when small electron-dense granules are also noticeable in the perioocytic space (Fig. 6C). At $48 \mathrm{~h}$ PBM, RER cisternae occupy most of the cytoplasm (Fig. 6D), while electron-dense plaques that increase in number and size distort the surface microvilli of the oocyte (Fig. 6D). Simultaneously, small electron-dense vesicles can be observed in the apical region of the follicular cells (Fig. 6D, insert). These vesicles seem to merge, forming large dense plaques at the inner side of the follicular cell membrane at $60 \mathrm{~h}$ PBM (Fig. 6E). At this time, the follicular cell cytoplasm already contains several vacuoles (Fig. $6 \mathrm{E})$. Degeneration of the follicular cells takes place during a process that occurs from $60 \mathrm{~h}$ PBM till approximately 84 h PBM, when the cells have completely degenerated (Fig. 6F). Oviposition occurs at approximately $90 \mathrm{~h} \mathrm{PBM} \mathrm{(87.39}$ \pm 5.46 , according to our observations) and concludes the gonotrophic cycle. 

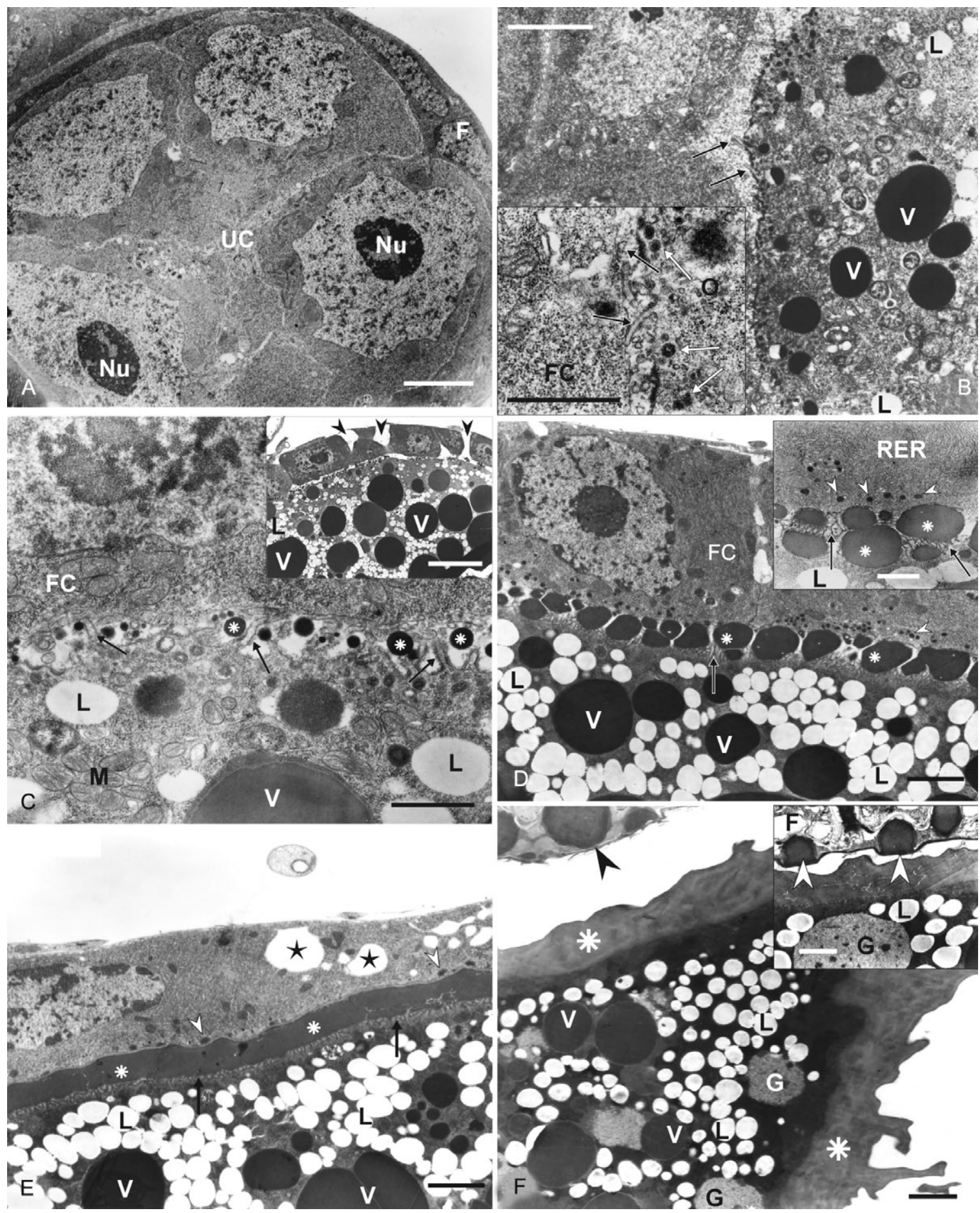

Fig. 6: electron micrographs of Culex quinquefasciatus ovarian follicle. A: before the blood meal, the primary follicle is constituted by undifferentiated cells (UC) surrounded by follicular epithelium (F). Nu: nucleolus. Bar: $3 \mu \mathrm{m}$; B: $12 \mathrm{~h}$ post-blood meal (PBM) follicle. Oocyte cytoplasm already presents vitellin granules (V) and lipid droplets (L). Microvilli (arrows) of oocyte membrane facilitate the nutrients uptake, evidenced by abundant vesicles in the oocyte apical cytoplasm (white arrows, insert). FC: follicular cell. Bar: $2 \mu \mathrm{m}$, insert: $1 \mu \mathrm{m}$. C: $24 \mathrm{~h}$ PBM follicle. Insert illustrate the great quantity of mitochondria (M), L and V inside the oocyte as well as the broad channels (arrowhead) in between the FCs. Electrondense granules (asterisks), putative endochorion precursors, fill the perioocytic space between microvilli (arrows). Bar: $1 \mu \mathrm{m}$, insert: $8 \mu \mathrm{m}$. D: $48 \mathrm{~h}$ PBM follicle. Well developed rough endoplasmic reticulum (RER) occupies almost the entire FC cytoplasm, while electrondense vesicles (arrowheads) are noticeable in the apical region. Perioocytic space is filled with chorionic plates (asterisks) that distort the microvilli (arrows) architecture. Bar: $2 \mu \mathrm{m}$, insert: $1 \mu \mathrm{m}$; E: $60 \mathrm{~h}$ PBM follicle. Fcs present several intracellular vacuoles (stars) suggesting the beginning of cell degeneration, however dense vesicles (arrowheads) are still noticeable in the apical region. Endochorion (asterisks) is almost completely formed squashing the oocyte microvilli (arrows). Bar: $2 \mu \mathrm{m}$; F: $84 \mathrm{~h} \mathrm{PBM}$ follicle. Besides L and V, oocyte now exhibits glycogen depots (G). Endochorion (asterisks) is now a continuous electrondense line. Electrondense plaques (arrowheads) suggestive of exochorion, protrude onto the apical membrane of the FC, totally degenerated. Bar: $2 \mu \mathrm{m}$, insert: $1 \mu \mathrm{m}$. 


\section{DISCUSSION}

As early as 1978, Atlas et al. suggested that the Culex pipiens fatigans yolk protein has a native molecular mass of $380 \mathrm{kDa}$, contains protein-bound phosphate, lipid and carbohydrate and is composed of two polypeptides of 80 and $160 \mathrm{kDa}$. Insect Vgs are synthesised as precursors that undergo processing as well as proteolytic cleavage into two or more subunits of smaller size (Dhadialla \& Raikhel 1990). Ae. aegypti Vg is also formed by two subunits of 200 and $65 \mathrm{kDa}$ (Raikhel 1987, Bose \& Raikhel 1988, Dhadialla \& Raikhel 1991). The difference in mass between the two mosquito Vgs is probably due to posttranslational modifications such as glycosylation (Bose \& Raikhel 1988). SDS-PAGE of fat body and haemolymph of $C x$. quinquefasciatus only allow to visualise the larger polypeptide of $\mathrm{Vg}$ (possibly the smaller is masked by the smaller polypeptide of lipophorin because both have nearly the same mass (AF Cardoso, unpublished observations). The molecular mass of the $\mathrm{Vg}$ polypeptides is consistent with the expected sizes listed in the annotation of the genome (XP_001857970.1). The amino acid sequence has a predicted mass of $241.609 \mathrm{kDa}$, while our studies indicate that $C x$. quinquefasciatus Vg has a molecular mass of $278 \mathrm{kDa}$. The difference is probably due to the posttranslational modifications that occur in the trophocyte. Trials using concanavalin-A confirmed that Cx. quinquefasciatus $\mathrm{Vg}$ is glycosylated (AF Cardoso, unpublished observations).

When analysing the similarities and differences between the oogenesis processes in $C x$. quinquefasciatus and $A e$. aegypti, the first thing that catches one's attention is the length of oogenesis and the oviposition time. In simultaneous experiments conducted under the same environmental conditions, Ae. aegypti females lay eggs sooner after a blood meal (63 \pm 1 h PBM) (F de Almeida, unpublished observations) than $C x$. quinquefasciatus females $(87.39 \pm 5.46 \mathrm{~h}$ PBM). The oviposition time that we determined for Ae. aegypti is in agreement with that communicated by Gomes et al. (2006). Morphological observations of the $C x$. quinquefasciatus fat bodies and ovaries prior to and during oogenesis were compared to those previously described for the better-studied Ae. aegypti.

In a general, the appearance of the fat body in female $C x$. quinquefasciatus mosquitoes is similar to that described for other mosquitoes. Four cell types have been described in arthropod fat bodies. Trophocytes are involved in intermediate metabolism and storage and the synthesis of Vg. Urocytes are engaged in the storage and secretion of uric acid. Mycetocytes are associated with the fat body in some insect species that have intracellular symbiotic microorganisms (Keeley 1978). Oenocytes are associated with the synthesis of hydrocarbons, precursors of cuticle lipids and pheromones in cockroaches (Fan et al. 2003).

The Cx. quinquefasciatus fat body is mainly composed of large trophocytes, but we also observed oenocyte-like cells. Unlike the description of Ae. aegypti oenocytes presented by Tadkowski et al. (1977), we failed to observe any change in these cells throughout the entire gonotrophic cycle.
When compared with other descriptions, trophocytes of the $C x$. quinquefasciatus fat body exhibit what appears to be a temporal discrepancy. A previtellogenic phase, in which the trophocytes become competent to synthesise $\mathrm{Vg}$, has been described in the studies conducted with Ae. aegypti. During this phase (the 1st 3 days after hatching), enlargement and activation of the nucleoli, proliferation of the ribosomes and RER, development of Golgi complexes and extensive invaginations of the plasma membrane occur (Raikhel \& Lea 1983). In Cx. quinquefasciatus, we did not observe this preparatory phase or activation of the biosynthetic machinery prior to a blood feeding. Moreover, we did not detect $\mathrm{Vg}$ synthesis or accumulation earlier than $12 \mathrm{~h}$ PBM. This physiological discrepancy, which is evidenced by morphological observations, may determine the chronological differences between the two mosquito species. Vg synthesis was observed by immunofluorescence at $3 \mathrm{~h}$ PBM in Ae. aegypti trophocytes (Raikhel \& Lea 1983) whereas in Cx. quinquefasciatus, Vg synthesis begins at approximately $12 \mathrm{~h}$ PBM as evidenced by immunoblotting and the presence of only a few, small RER cisternae. In $C x$. quinquefasciatus, the emergence of electron-dense vesicles and granules from the Golgi complex of the trophocytes was not observed earlier than $24 \mathrm{~h}$ PBM.

In $A$ e. aegypti, the trophocyte ultrastructure indicates a decline in Vg synthesis at 30-40 h PBM when the RER and Golgi complex are reduced and the secretion granules disappear (Behan \& Hagedorn 1978, Raikhel \& Lea 1983). In Cx. quinquefasciatus, the reduction of the biosynthetic organelles begins between 48-72 h PBM. Only at $84 \mathrm{~h}$ PBM do the trophocytes of Cx. quinquefasciatus return to the appearance that they had before the blood meal, $14 \mathrm{~h}$ later than the time described for Ae. aegypti (Behan \& Hagedorn 1978).

The general structure of the $C x$. quinquefasciatus ovaries is similar to that of other mosquitoes. Nonetheless, we observed morphological differences and some discrepancies in the temporal patterns of development between $C x$. quinquefasciatus and Ae. aegypti. Several studies observed that while Ae. aegypti was still in its previtellogenic phase, oocyte differentiation was initiated and it became distinguishable from the other cells due to its smaller nucleus with a prominent nucleolus and the presence of lipid inclusions in its cytoplasm (Roth \& Porter 1964, Raikhel \& Lea 1983, Clements \& Boocock 1984). This initial differentiation does not occur in pre-engorged $C x$. quinquefasciatus, whose ovarian follicles consist of a set of eight undifferentiated cells before the first blood meal.

The uptake of $\mathrm{Vg}$ by oocytes is a receptor-mediated, clathrin-dependent endocytosis process (Roth \& Porter 1964, Raikhel 1984, Raikhel \& Dhadialla 1992). Accordingly, during the vitellogenic phase, coated vesicles are visible in the peripheral cytoplasm of $C x$. quinquefasciatus oocytes.

Lipid and carbohydrates also accumulate in the developing oocytes. While lipid storage occurs with $\mathrm{Vg}$ uptake, carbohydrate accumulation is only evident at the 
end of oogenesis. Briegel et al. (2003) described that in Ae. aegypti the deposition of glycogen is delayed when compared with vitellin and lipids. Van Handel (1992, 1993) also described a delayed accumulation of glycogen in $C x$. quinquefasciatus and showed that glycogen is the only reserve that will be used by the mature oocytes to maintain their own integrity during the period between the chorionation and the oviposition.

Mosquito follicular cells are responsible for the synthesis and secretion of the eggshell components into the perioocytic space (Raikhel \& Lea 1991, Lucantoni et al. 2006). The eggshell is an important structure that protects the oocyte and/or the developing embryo from a series of environmental hazards (Yao \& Li 2003). Eggshell protein synthesis and secretion by the follicular cells is hormonally controlled by 20-hydroxyecdysone (Raikhel \& Lea 1991, Edwards et al. 1998) and occurs in two phases, which results in two distinct layers called the endochorion and exochorion (Hinton 1968, Monnerat et al. 1999).

In Cx. quinquefasciatus, the follicular cells showed no morphological indications of synthetic activity before the engorgement and throughout the first $18 \mathrm{~h}$ PBM. Morphological changes begin to occur at approximately $24 \mathrm{~h}$ PBM with an increase in RER cisternae. At $48 \mathrm{~h}$ PBM, electron-dense plaques accumulate in the perioocytic space between the follicular cells and the oocyte and may represent the secretion of components of the endochorion layer. Later, at $60 \mathrm{~h}$ PBM, small electrondense vesicles can be observed in the apical region of the follicular cells of $C x$. quinquefasciatus. These vesicles seem to merge and form large dense granules, which are presumably precursors of the exochorion. Degeneration of the follicular cells occurs in a process that spans from $60 \mathrm{~h}$ PBM to approximately $84 \mathrm{~h}$ PBM, at which time they are completely degenerated. Follicular cell degeneration determines the end of the oogenesis process.

In conclusion, we have presented a morphological description of two organs involved in the process of egg formation in the mosquito $C x$. quinquefasciatus, the fat body and the ovaries. We have also correlated the morphology with the protein profiles of the both organs. Comparisons between our observations and similar descriptions previously presented for the mosquito Ae. aegypti revealed a number of similarities and differences. With this study, we hope to contribute further knowledge of the physiology of this important, cosmopolitan and urban plague, the mosquito Cx. quinquefasciatus.

\section{ACKNOWLEDGEMENTS}

To Carlos E Winter, for the interesting suggestions during the development of this project, to Manoel Aparecido Peres, by technical support, and to Osvaldo Marinotti, for the critical evaluation of manuscript.

\section{REFERENCES}

Atlas SJ, Roth TF, Falcone AJ 1978. Purification and partial characterization of Culex pipiens fatigans yolk protein. Insect Biochem $8: 111-115$.

Attardo GM, Hansen IA, Raikhel AS 2005. Nutritional regulation of vitellogenesis in mosquitoes: implications for anautogeny. Insect Biochem Mol Biol 35: 661-675.
Behan M, Hagedorn HH 1978. Ultrastructural changes in the fat body of adult female Aedes aegypti in relationship to vitellogenin synthesis. Cell Tissue Res 186: 499-506.

Bose SG, Raikhel AS 1988. Mosquito vitellogenin subunits originate from a common precursor. Biochem Biophys Res Commun 155: 436-442.

Bradford MM 1976. A rapid and sensitive method for the quantitation of microgram quantities of protein utilizing the principle of protein-dye binding. Anal Biochem 72: 248-254.

Briegel H, Gut T, Lea AO 2003. Sequential deposition of yolk components during oogenesis in an insect, Aedes aegypti (Diptera: Culicidae). J Insect Physiol 49: 249-260.

Chapman RF 1998. The insects: structure and function, 4th ed., Cambridge University Press, Cambridge, 770 pp.

Clements AN 1992. The biology of mosquitoes. Development, nutrition and reproduction, 1th ed., Chapman and Hall press, London, $509 \mathrm{pp}$.

Clements AN, Boocock MR 1984. Ovarian development in mosquitoes: stages of growth and arrest and follicular resorption. Physiol Entomol 9: 1-8.

da Cunha Sais T, de Moraes RM, Ribolla PE, de Bianchi AG, Marinotti O, Bijovsky AT 2003. Morphological aspects of Culex quinquefasciatus salivary glands. Arthropod Struct Dev 32: 219-226.

de Bianchi AG, Coutinho M, Pereira SD, Marinotti O, Targa HJ 1985. Vitellogenin and vitellin of Musca domestica - Quantification and synthesis by fat-bodies and ovaries. Insect Biochem 15: 77-84.

Dhadialla TS, Raikhel AS 1990. Biosynthesis of mosquito vitellogenin. J Biol Chem 265: 9924-9933.

Dhadialla TS, Raikhel AS 1991. Binding of vitellogenin to membranes isolated from mosquito ovaries. Arch Insect Biochem Physiol 18: 55-70.

Edwards MJ, Severson DW, Hagedorn HH 1998. Vitelline envelope genes of yellow fever mosquito, Aedes aegypti. Insect Biochem Mol Biol 28: 915-925.

Fallon AM, Hagedorn HH, Wyatt GR, Laufer H 1974. Activation of vitellogenin synthesis in the mosquito Aedes aegypti by ecdysone. J Insect Physiol 20: 1815-1823.

Fan YL, Zurek L, Dykstra MJ, Schal C 2003. Hydrocarbon synthesis by enzymatically dissociated oenocytes of the abdominal integument of the German Cockroach, Blaftella germanica. Naturwissenschaften 90: 121-126.

Gaunt MW, Sall AA, Lamballerie XD, Falconar AKI, Dzhivanian TI, Gould EA 2001. Phylogenetic relationships of flaviviruses correlate with their epidemiology, disease association and biogeography. J Gen Virol 82: 1867-1876.

Gomes AS, de Sa Sciavico CJ, Eiras AE 2006. Periodicity of oviposition of females of Aedes aegypti (Linnaeus, 1762) (Diptera: Culicidae) in laboratory and field. Rev Soc Bras Med Trop 39: 327-332.

Hagedorn HH, Fallon A, Laufer H 1973. Vitellogenin synthesis by the fat body of the mosquito Aedes aegypti: evidence of transcriptional control. Dev Biol 31: 285-294.

Hagedorn HH, Judson CL 1972. Purification and site of synthesis of Aedes aegypti yolk proteins. J Exp Zool 182: 367-377.

Hamer GL, Kitron UD, Brawn JD, Loss SR, Ruiz MO, Goldberg TL, Walker ED 2008. Culex pipiens (Diptera: Culicidae): a bridge vector of West Nile virus to humans. J Med Entomol 45: 125-128.

Hinton HE 1968. Structure and protective devices of the egg of the mosquito Culex pipiens. J Insect Physiol 14: 145-161. 
Keeley LL 1978. Endocrine regulation of fat-body development and function. Annu Rev Entomol 23: 329-352.

Laemmli UK 1970. Cleavage of structural proteins during the assembly of the head of bacteriophage T4. Nature 227: 680-685.

Lucantoni L, Giusti F, Cristofaro M, Pasqualini L, Esposito F, Lupetti P, Habluetzel A 2006. Effects of a neem extract on blood feeding, oviposition and oocyte ultrastructure in Anopheles stephensi Liston (Diptera: Culicidae). Tissue Cell 38: 361-371.

Malafronte RS, Calvo E, James AA, Marinotti O 2003. The major salivary gland antigens of Culex quinquefasciatus are D7-related proteins. Insect Biochem Mol Biol 33: 63-71.

Monnerat AT, Soares MJ, Lima JBP, Rosa-Freitas MG, Valle D 1999. Anopheles albitarsis eggs: ultrastructural analysis of chorion layers after permeabilization. J Insect Physiol 45: 915-922.

Okuda K, de Almeida F, Mortara RA, Krieger H, Marinotti O, Bijovsky AT 2007. Cell death and regeneration in the midgut of the mosquito, Culex quinquefasciatus. J Insect Physiol 53: $1307-1315$.

Okuda K, de Souza Caroci A, Ribolla PE, de Bianchi AG, Bijovsky AT 2002. Functional morphology of adult female Culex quinquefasciatus midgut during blood digestion. Tissue Cell 34: 210-219.

Okuda K, Esteva M, Segura EL, Bijovsy AT 1999. The cytostome of Trypanosoma cruzi epimastigotes is associated with the flagellar complex. Exp Parasitol 92: 223-231.

Raikhel AS 1984. The accumulative pathway of vitellogenin in the mosquito oocyte: a high-resolution immuno and cytochemical study. J Ultrastruct Res 87: 285-302.

Raikhel AS 1986. Lysosomes in the cessation of vitellogenin secretion by the mosquito fat body; selective degradation of Golgi complexes and secretory granules. Tissue Cell 18: 125-142.

Raikhel AS 1987. Monoclonal antibodies as probes for processing of the mosquito yolk protein; a high-resolution immunolocalization of secretory and accumulative pathways. Tissue Cell 19: 515-529.

Raikhel AS, Dhadialla TS 1992. Accumulation of yolk proteins in insect oocytes. Ann Rev Entomol 37: 217-251.

Raikhel AS, Lea AO 1983. Previtellogenic development and vitello- genin synthesis in the fat body of a mosquito: an ultrastructural and immunocytochemical study. Tissue Cell 15: 281-300.

Raikhel AS, Lea AO 1991. Control of follicular epithelium development and vitelline envelope formation in the mosquito; role of juvenile hormone and 20-hydroxyecdysone. Tissue Cell 23: 577-591.

Ribeiro JM, Charlab R, Pham VM, Garfield M, Valenzuela JG 2004. An insight into the salivary transcriptome and proteome of the adult female mosquito Culex pipiens quinquefasciatus. Insect Biochem Mol Biol 34: 543-563.

Roth TF, Porter KR 1964. Yolk protein uptake in the oocyte of the mosquito Aedes aegypti. L. J Cell Biol 20: 313-332.

Sappington TW, Raikhel AS 1998. Molecular characteristics of insect vitellogenins and vitellogenin receptors. Insect Biochem Mol Biol 28: 277-300.

Snigirevskaya ES, Hays AR, Raikhel AS 1997. Secretory and internalization pathways of mosquito yolk protein precursors. Cell Tissue Res 290: 129-142.

Tadkowski TM, Jones JC 1978. Endogenous synthesis of lipid yolk in mosquito oocytes. Experientia 34: 627-627.

Tadkowski TM, Jones JC, Firman J 1977. The fine structure of the imaginal oenocytes of Aedes aegypti (Diptera: Culicidae). Annals Entom Soc Am 70: 837-840.

Vaidyanathan R, Scott TW 2007. Geographic variation in vector competence for West Nile virus in the Culex pipiens (Diptera: Culicidae) complex in California. Vector-Borne Zoon Dis 7: 193-198.

Van Essen PHA, Kemme JA, Ritchie SA, Kay BH 1994. Differential responses of Aedes and Culex mosquitoes to octenol or light in combination with carbon dioxide in Queensland, Australia. Med Vet Entomol 8: 63-67.

Van Handel E 1992. Postvitellogenic metabolism of the mosquito (Culex quinquefasciatus) ovary. J Insect Physiol 38: 75-79.

Van Handel E 1993. Fuel metabolism of the mosquito (Culex quinquefasciatus) embryo. J Insect Physiol 39: 831-833.

Yao R, Li J 2003. Towards global analysis of mosquito chorion proteins through sequential extraction, two-dimensional electrophoresis and mass spectrometry. Proteomics 3: 2036-2043. 